\title{
Priority of lymph node dissection for proximal gastric cancer invading the greater curvature
}

\author{
Yukio Maezawa $^{1,2} \cdot$ Toru Aoyama $^{1,2} \cdot$ Takanobu Yamada $^{1,2} \cdot$ Kazuki Kano $^{1,2}$. \\ Tsutomu Hayashi ${ }^{2} \cdot$ Tsutomu Sato $^{2} \cdot$ Takashi Oshima $^{2} \cdot$ Yasushi Rino $^{2}$. \\ Munetaka Masuda $^{2} \cdot$ Takashi Ogata $^{1} \cdot$ Haruhiko Cho ${ }^{1,2} \cdot$ Takaki Yoshikawa $^{1,2}$
}

Received: 19 May 2017 / Accepted: 23 October 2017 / Published online: 8 November 2017

(c) The International Gastric Cancer Association and The Japanese Gastric Cancer Association 2017

\begin{abstract}
Background The therapeutic efficacy of dissection of the splenic hilar lymph nodes (\#10) has not been fully evaluated in locally advanced proximal gastric cancer (LAGC) invading the greater curvature of the stomach.

Methods Patients with LAGC invading the greater curvature who underwent D2 total gastrectomy with splenectomy between January 2000 and May 2015 were retrospectively examined. The therapeutic value index was calculated by multiplying the metastatic rate of a station and the 5-year survival of patients with metastasis to that station; the metastatic rate and the index of each lymph node station were then compared.

Results In total, 82 patients were eligible for the present study. The most frequent metastatic node was \#3, followed by \#1, \#4d and \#7, \#2, \#4sa and \#10, and \#4sb and \#9. These lymph nodes had a metastatic rate of more than $10 \%$. The node station with the highest index was \#3, followed by \#7, $\# 4 \mathrm{~d}$, \#1, \#4sb, \#4sa, \#2, and \#9. The index of \#10 was lower, but exceeded those of \#8a and \#11p.

Conclusions The metastatic rate of \#10 was relatively high at $13 \%$, and the therapeutic value index was as high as those of other suprapancreatic nodes. D2 dissection for proximal cancer located in the greater curvature should include removal of node \#10.
\end{abstract}

Yukio Maezawa and Toru Aoyama contributed equally

Takaki Yoshikawa

yoshikawat@kcch.jp

1 Department of Gastrointestinal Surgery, Kanagawa Cancer Center, 2-3-2 Nakao, Asahi-ku, Yokohama 241-8515, Japan

2 Department of Surgery, Yokohama City University, Yokohama, Japan
Keywords Gastric cancer · Greater curvature · Therapeutic index $\cdot$ Metastatic rate

\section{Introduction}

A total of 951,600 new cases and 723,100 deaths caused by gastric cancer occurred worldwide in 2012 [1]. D2 gastrectomy is now a globally accepted surgical procedure for resectable disease. The original D2 dissection for cancer of the upper third of the stomach required splenectomy for advanced gastric cancer to dissect splenic hilar lymph nodes (\#10) regardless of the circumferential location of the tumor, but whether to observe this rule rigorously has long been a matter of controversy in Japan, whereas splenectomy had been discarded in the Western world for reasons of high mortality [2]. Recently, the Japan Clinical Oncology Group phase III study (JCOG0110) clearly confirmed the survival noninferiority of spleen-preserving surgery against splenectomy for proximal advanced gastric cancer not invading the greater curvature [3]. Therefore, spleen-preserving D2 gastrectomy is a standard procedure for this population. However, complete dissection of the nodes around the splenic hilum is rendered extremely difficult by spleen-preserving D2 gastrectomy. It is still unclear whether \#10 had no impact on survival or had a survival benefit that was canceled by splenectomy.

On the other hand, theoretical lymphatic flow from the upper part of the greater curvature goes into \#10 [4, 5]. Therefore, tumors invading the greater curvature had been excluded in the entry criteria of the JCOG0110 trial. Because the results of the JCOG0110 trial are not applicable to tumors invading the greater curvature, the recommendation by the Japanese guidelines to perform splenectomy for meticulous dissection of \#10 in this population remains 
valid. However, the therapeutic efficacy of splenic hilar nodal dissection has not been fully evaluated. If the therapeutic effect was less than for other regional lymph node stations, splenectomy may be reconsidered for these tumors.

Based on these considerations, we evaluated the therapeutic efficacy of \#10 by comparing that of other regional nodes in patients who underwent total gastrectomy with splenectomy for locally advanced proximal gastric cancer invading the greater curvature.

\section{Patients and methods}

\section{Patients}

The patients were retrospectively selected from the medical records of consecutive patients who underwent gastrectomy at Kanagawa Cancer Center from January 2000 to May 2015, according to the following criteria: (1) histologically proven gastric adenocarcinoma diagnosed as pathological T2-T4, invading the upper third of the stomach and greater curvature [6]; and (2) underwent D2 total gastrectomy with splenectomy and achieved $\mathrm{R} 0$ resection. The resected specimens were examined pathologically and evaluated according to the Japanese classification of gastric carcinoma (third English edition) [6].

\section{Surgical procedure}

Total gastrectomy with splenectomy was performed with full mobilization of the body and tail of the pancreas and spleen. The lymph nodes along the splenic artery were completely dissected, and the spleen was removed en bloc together with \#10. The reconstruction method was at the surgeon's discretion.

\section{Statistical analyses}

To evaluate the efficacy of nodal dissection, we adopted a method using the therapeutic value index introduced by Sasako et al. in 1995 [7]. The index was calculated by multiplying the frequency of metastasis to the station and the 5-year survival rate of patients with metastasis to that station. Nodes were picked up from the specimen and were assigned to the appropriate 'station' according to the Japanese classification of gastric carcinoma (third English edition). Nodes found at each station were labeled and sent for histological examination.

Overall survival (OS) was defined as the period from the date of surgery to the date of death from any cause. The data of the patients who did not experience an event were censored on the date of the final observation. The survival data were obtained from hospital records or from the city registry system. This study was approved by the Institutional Review Board (IRB) of the Kanagawa Cancer Center (2016 epidemiologic study-22).

\section{Results}

\section{Patient background}

Among the 162 patients who underwent total gastrectomy for proximal gastric cancer invading the greater curvature, a total of 82 patients were eligible for the present study. Figure 1 showed the consort diagram of the present study. Patient age ranged from 29 to 83 years (median, 65 years); 56 were men and 26 were women. Forty patients received the adjuvant treatment. The patients received S-1 adjuvant chemotherapy as a clinical practice after July 2006 when the patients were diagnosed with stage II/III except T1N2-3/T3N0 [8]. Table 1 summarizes the patient demographics.

\section{Efficacy of dissection}

The metastatic rate, 5-year survival rates, and therapeutic index are shown in Table 2. The lymph node stations with the highest incidence of metastasis were \#3, followed by \#1, \#4d and \#7, \#2, \#4sa and \#10, and \#4sb and \#9. These lymph node stations had a metastatic rate greater than $10 \%$. The node station with the highest index was \#3, followed by \#7, \#4d, \#1, \#4sb, \#4sa, \#2, and \#9. The index of \#10 was lower, but exceeded those of \#8a and \#11p. No patient survived for more than 5 years when the cancer metastasized to \#11d or \#12a.

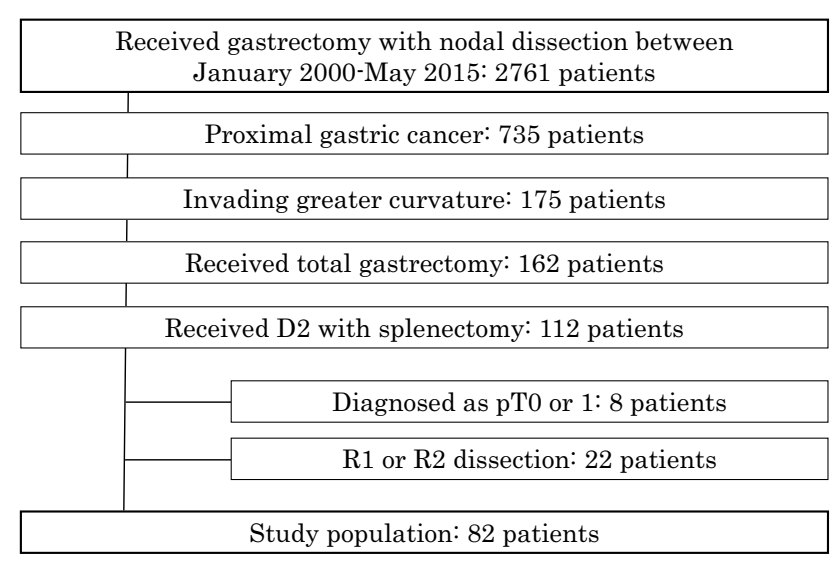

Fig. 1 Flow diagram of the 2761 patients who underwent gastrectomy for gastric cancer between January 2000 and May 2015 
Table 1 Patient and tumor characteristics $(n=82)$

\begin{tabular}{ll}
\hline Characteristics & Value \\
\hline Age (years), median (range) & $65(29-83)$ \\
Gender & \\
Male & $56(68 \%)$ \\
Female & $26(32 \%)$ \\
BMI, median (range) & $22.8(15.4-29.4)$ \\
ASA-PS & \\
1 & $32(39 \%)$ \\
2 & $50(61 \%)$ \\
Main tumor location & \\
Upper third & $64(78 \%)$ \\
Middle third & $18(22 \%)$ \\
Histological type & \\
Intestinal & $30(37 \%)$ \\
Diffuse & $52(63 \%)$ \\
Macroscopic type & \\
Non-type 4 & $66(80 \%)$ \\
Type 4 & $16(20 \%)$ \\
Tumor diameter (mm), median (range) & $61(18-200)$ \\
Pathological T factor & \\
pT2 & $24(29 \%)$ \\
pT3 & $51(62 \%)$ \\
pT4 & \\
Pathological N factor & $16(35 \%)$ \\
pN0 & $10(12 \%)$ \\
pN1 & $27(21 \%)$ \\
pN2 & \\
pN3 & \\
Adjuvant chemotherapy & \\
No & \\
Yes & \\
\hline
\end{tabular}

$B M I$ body mass index, ASA-PS ASA physical status classification

\section{Discussion}

The present study evaluated the therapeutic efficacy of each lymph node station dissected in locally advanced proximal gastric cancer invading the greater curvature. We found that the metastatic rate of the splenic hilar node was greater than $10 \%$, which was comparable to the perigastric nodes of \#4sa and \#4sb, and that the priority of splenic hilar node dissection was higher than that of lymph nodes along the common hepatic artery and the splenic artery, suggesting that the splenic hilar node had a value to be involved in a part of D2.

The metastatic rate of \#10 was $13.4 \%$ in the present study. Previously, Sasako et al. reported that the metastatic rate of \#10 in the tumors located in the upper third of the stomach was about 18\% [7]. Their study included all proximal tumors regardless of the invasion to the greater curvature. On the other hand, the metastatic rate of \#10 was low (only 2.4\%)
Table 2 Details of metastatic rate, overall survival (OS), and therapeutic value index within the regional lymph nodes

\begin{tabular}{lrll}
\hline Station no. & $\begin{array}{l}\text { Metastatic rate } \\
(\%)\end{array}$ & 5 -year OS (\%) & $\begin{array}{l}\text { Therapeutic } \\
\text { value index }\end{array}$ \\
\hline 1 & 23.2 & 33.6 & 7.79 \\
2 & 19.5 & 30.0 & 5.85 \\
3 & 51.2 & 59.7 & 30.6 \\
$4 \mathrm{sa}$ & 13.4 & 50.0 & 6.70 \\
$4 \mathrm{sb}$ & 11.0 & 62.5 & 6.88 \\
$4 \mathrm{~d}$ & 22.0 & 41.3 & 9.09 \\
$5^{\mathrm{a}}$ & 1.2 & - & - \\
6 & 9.8 & 14.6 & 1.43 \\
7 & 22.0 & 48.9 & 10.8 \\
$8 \mathrm{a}$ & 4.9 & 50.0 & 2.45 \\
9 & 11.0 & 50.0 & 5.50 \\
10 & 13.4 & 30.0 & 4.02 \\
$11 \mathrm{p}$ & 6.1 & 40.0 & 2.44 \\
$11 \mathrm{~d}$ & 2.4 & 0.0 & 0.00 \\
$12 \mathrm{a}$ & 1.2 & 0.0 & 0.00 \\
\hline
\end{tabular}

${ }^{\text {a }}$ There was only one case of metastasis positive in $\# 5$ and the case is censored; OS is not calculated

in the JCOG0110 trial, which excluded the tumors invading the greater curvature [3]. Thus, the metastasis to \#10 is almost limited to gastric cancers that are located at the greater curvature. Interestingly, the high metastatic rate of \#10 was almost similar to that of \#4sa or \#4sb in the present study, which would support that the cancer metastasized following the theoretical lymphatic flow from the greater curvature of the stomach to the splenic hilum. To confirm whether dissection of \#10 has therapeutic value, we calculated the therapeutic index by multiplying the metastatic rate with the 5-year survival rate.

In this study, the therapeutic value index of \#10 came next to the perigastric nodes and \#9 and was substantially higher than those of \#8a, \#11p, and \#11d, all of which had been essential components of $\mathrm{D} 2$ dissection. This finding suggests that the priority of \#10 is next to that of perigastric nodes and is higher than that of the lymph nodes along the splenic artery. Previously, Sasako et al. calculated the therapeutic value index in all tumors located at the upper stomach [7]. In their study, the highest priority lymph node stations were the perigastric nodes of \#3, \#1, \#7, and \#2, followed by \#10 and \#9. The index of \#10 was higher than that of \#8 and \#11. Although their study did include the tumors not invading the greater curvature, the order of the nodal index in Sasako's study was almost concordant with the present study. Given these data, we recommend that \#10 be treated alongside other suprapancreatic or second-tier lymph nodes in the D2 dissection for this cohort.

There are several limitations associated with this study. The first issue is a selection bias of the present cohort. As 
shown in the consort, not all the patients with proximal cancer invading the greater curvature received splenectomy. Because of the retrospective nature of the study, we could not identify the reasons for spleen preservation although total gastrectomy with splenectomy had been the standard for this population throughout the study period. Thus, the present cohort had some unknown selection bias that could have affected the present results. Second, there were some variations in adjuvant therapy after surgery. Some patients did not receive adjuvant therapy. The results could have been different in another cohort in which S-1 adjuvant chemotherapy was selected.

In conclusion, the present study showed that splenic hilar lymph nodes had a high metastatic rate, more than $10 \%$, and had a high therapeutic index exceeding the suprapancreatic lymph node stations. The splenic hilar nodes deserve to be a part of D2 lymphadenectomy for proximal tumors invading the greater curvature, although this may not necessarily involve splenectomy.

Acknowledgements The authors express their sincere gratitude to Ms. Natsumi Sato and Ms. Rika Takahashi for their excellent data management in this study.

\section{Compliance with ethical standards}

Conflict of interest The authors declare no conflicts of interest in association with the present study.

Human rights statement The study data and informed consent were obtained in accordance with the Declaration of Helsinki and were approved by the Ethics Review Board of Kanagawa Cancer Center.
Informed consent Informed consent or substitute for it was obtained from all patients for being included in the study.

\section{References}

1. Torre LA, Bray F, Siegel RL, Ferlay J, Lortet-Tieulent J, Jemal A. Global cancer statistics, 2012. CA Cancer J Clin. 2015;65(2):87-108.

2. Cuschieri A, Weeden S, Fielding J, Bancewicz J, Craven J, Joypaul $\mathrm{V}$, et al. Patient survival after D1 and D2 resections for gastric cancer: long-term results of the MRC randomized surgical trial. Surgical Co-operative Group. Br J Cancer. 1999;79(9-10):1522-30.

3. Sano T, Sasako M, Mizusawa J, Yamamoto S, Katai H, Yoshikawa $\mathrm{T}$, et al. Randomized controlled trial to evaluate splenectomy in total gastrectomy for proximal gastric carcinoma. Ann Surg. 2017;265:277-83.

4. Maruyama K, Okabayashi K, Kinoshita T. Progress in gastric cancer surgery in Japan and its limits of radicality. World J Surg. 1987;11(4):418-25.

5. Takahashi T, Sawai K, Hagiwara A, Takahashi S, Seiki K, Tokuda $\mathrm{H}$. Type-oriented therapy for gastric cancer effective for lymph node metastasis: management of lymph node metastasis using activated carbon particles adsorbing an anticancer agent. Semin Surg Oncol. 1991;7(6):378-83.

6. Japanese Gastric Cancer Association. Japanese classification of gastric carcinoma, 3rd English edition. Gastric Cancer. 2011;14(2):101-12.

7. Sasako MMP, Kinoshita T, Maruyama K. New method to evaluate the therapeutic value of lymph node dissection for gastric cancer. Br J Surg. 1995;82(3):6.

8. Sakuramoto S, Sasako M, Yamaguchi T, Kinoshita T, Fujii $\mathrm{M}$, Nashimoto A, et al. Adjuvant chemotherapy for gastric cancer with S-1, an oral fluoropyrimidine. N Engl J Med. 2007;357(18):1810-20. 